\title{
Achieving AWGN Capacity Under Stochastic Energy Harvesting
}

\author{
Omur Ozel, Student Member, IEEE, and Sennur Ulukus, Member, IEEE
}

\begin{abstract}
In energy harvesting communication systems, an exogenous recharge process supplies energy necessary for data transmission and the arriving energy can be buffered in a battery before consumption. We determine the information-theoretic capacity of the classical additive white Gaussian noise (AWGN) channel with an energy harvesting transmitter with an unlimited sized battery. As the energy arrives randomly and can be saved in the battery, codewords must obey cumulative stochastic energy constraints. We show that the capacity of the AWGN channel with such stochastic channel input constraints is equal to the capacity with an average power constraint equal to the average recharge rate. We provide two capacity achieving schemes: save-and-transmit and best-effort-transmit. In the save-and-transmit scheme, the transmitter collects energy in a saving phase of proper duration that guarantees that there will be no energy shortages during the transmission of code symbols. In the best-effort-transmit scheme, the transmission starts right away without an initial saving period, and the transmitter sends a code symbol if there is sufficient energy in the battery, and a zero symbol otherwise. Finally, we consider a system in which the average recharge rate is time varying in a larger time scale and derive the optimal offline power policy that maximizes the average throughput, by using majorization theory.
\end{abstract}

Index Terms-Additive white Gaussian noise (AWGN) channel, energy harvesting, offline power management, Shannon capacity.

\section{INTRODUCTION}

I $\mathrm{N}$ this paper, we analyze point-to-point communication of energy harvesting nodes from an information-theoretic perspective. We focus on wireless networking applications where nodes (e.g., sensors nodes) can harvest energy from nature through various different sources, such as solar cells, vibration absorption devices, water mills, thermoelectric generators, microbial fuel cells, etc. In such systems, energy that becomes available for data transmission can be modeled as an exogenous recharge process. Therefore, unlike traditional battery-powered systems, energy is not a deterministic quantity in these systems, but is a random process which varies stochastically in time

Manuscript received December 15, 2010; revised May 22, 2012 and June 03, 2012; accepted June 04, 2012. Date of publication September 11, 2012; date of current version September 11, 2012. This work was supported by the National Science Foundation under Grants CCF 04-47613, CCF 05-14846, CNS 07-16311, CCF 07-29127, and CNS 09-64632. This paper was presented in part at the International Workshop on Green Wireless (W-GREEN) at the 2010 IEEE International Symposium on Personal, Indoor, and Mobile Radio Communications.

The authors are with the Department of Electrical and Computer Engineering, University of Maryland, College Park, MD 20742 USA (e-mail: omur@umd. edu; ulukus@umd.edu).

Communicated by A. Lozano, Associate Editor for Communications.

Color versions of one or more of the figures in this paper are available online at http://ieeexplore.ieee.org.

Digital Object Identifier 10.1109/TIT.2012.2204389 at a scale on the order of symbol duration. Transmission can be interrupted due to lack of energy in the battery. On the other hand, excess energy can be buffered in the battery before consumption for transmission. This model requires a major shift in terms of the power constraint imposed on the channel input compared to those in the existing literature.

To illustrate, in information-theoretic approaches, there are two widely used input constraints on the channel inputs of continuous-alphabet channels: average power constraint and amplitude constraint. If the input is average power constrained, then any codeword should be such that while each symbol can take any real value, the average power of the entire codeword should be no more than the power constraint. On the other hand, if the input is amplitude constrained, then every code symbol should be less than the constraint in amplitude. It is clear that in an energy harvesting model, the constraint imposed on the channel input is different than these constraints, in that, while code symbols are instantaneously amplitude constrained, energy can be saved in the battery for later use. This amounts to an unprecedented input constraint on the channel input. In this context, the main goal of this paper is to investigate the impact of stochastic energy arrivals on the achievable communication rates in an information-theoretic framework. In particular, we augment an energy buffer to the classical additive white Gaussian noise (AWGN) system and study information-theoretically achievable rates.

First, we consider the setting where energy arrives at the transmitter as a discrete-time stochastic process, and unused energy is saved in a battery of unlimited size. The energy arrival (or recharge) process has the same discrete time index as the channel use. Therefore, the energy in the battery is updated as follows. First, it is increased by the energy arrival and then it is decreased by the energy of the transmitted code symbol. The problem is posed as the design of a codebook that complies with instantaneous energy constraints at each channel use. The channel input in each channel use is constrained by the amount of energy in the battery, which evolves stochastically throughout the communication. Therefore, this model generalizes classical deterministic amplitude constraint on the channel input. The recharge process together with the past code symbols determine the allowable range of inputs in each channel use. We start with showing that the capacity of the AWGN channel with an average power constraint equal to the average recharge rate is an upper bound for the capacity in the energy harvesting system. Then, we develop the save-and-transmit scheme that achieves this upper bound and hence the capacity. In the save-and-transmit scheme, zero code symbols are sent in a portion of the total block length, which becomes negligible as the block length gets large. The goal of this portion of the total block length where 
no signal is transmitted is to save energy to ensure that there will always be sufficient amount of energy to transmit the remaining code symbols, with probability approaching one. Next, we provide an alternative capacity achieving scheme termed the best-effort-transmit scheme. In this scheme, whenever available energy in the battery is sufficient to send the code symbol, it is put to the channel, while a zero symbol is put to the channel if there is not enough energy in the battery. This leads to a mismatch between the encoder and the decoder in the sense that some of the code symbols in the codeword are replaced with zeros. However, we show that the mismatch can be made negligible, and therefore, this scheme can achieve rates arbitrarily close to the capacity.

Second, we address a typical behavior in certain energy harvesting sensors, such as solar-powered sensors, where the recharge process is nonergodic and nonstationary. In this case, we assume that the average recharging rate is not a constant in time, but rather fosters time variation in a scale much larger than the time scale in which communication takes place. Accordingly, the recharge process has time-varying mean in sufficiently long time to which we refer to as a frame. Using tools from majorization theory, we derive the optimal offline power management policy for maximum average throughput in a finite number of frames, and provide a geometric interpretation for the resulting optimal policy.

\section{A. Related Literature}

Information-theoretic study of communication in energy harvesting systems has been recently initiated in [1]. There have been many motivating works in the networking literature. In [2], Lei et al. address replenishment in one hop transmission. Formulating transmission strategy as a Markov decision process, Lei et al. [2] use dynamic programming techniques for optimization of the transmission policy under replenishment. In [3], Gatzianas et al. extend classical wireless network scheduling results to a network with users having rechargeable batteries. Each battery is considered as an energy queue, and data and energy queues are simultaneously updated where interaction of these queues are determined by a rate versus power relationship. Stability of data queues is studied using Lyapunov techniques. A back pressure algorithm is proposed that takes both data and energy queues into consideration and it is shown to achieve the stability region of the average power-constrained system as the battery capacity goes to infinity. In [4] and [5], in a similar energy harvesting setting, a dynamic power management policy is proposed and is shown to stabilize the data queues. In each frame, energy spent is equal to the average recharge rate. Moreover, under a linear approximation, some delay-optimal schemes are proposed. In [6] and [7], optimal packet scheduling that minimizes the transmission completion time has been derived. In [8], energy allocation is optimized in a slotted system using dynamic programming techniques. In [9], we provided continuous time optimal policies for energy harvesting nodes in fading channels. Tutuncuoglu and Yener [10] extend [6], [7] to a setting with finite battery sizes, and we extend [6], [7] to a broadcast setting in [11] independently and concurrently with [12].

The capacity of scalar AWGN channel has been extensively studied in the literature under different constraints on the input

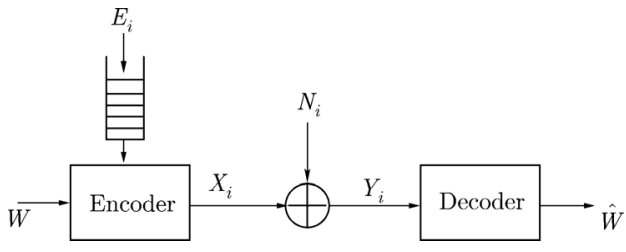

Fig. 1. AWGN channel with random energy arrivals.

signal. Average power (or the second moment) constraint on the input yields the well-known result that the capacity achieving input distribution is Gaussian with variance equal to the power constraint. Smith [13] considers amplitude constraints in addition to average power constraints and concludes that the capacity achieving input density function has all the mass distributed over finite number of points in the real line. Moreover, Shamai and Bar-David [14] extend Smith's result to amplitude-constrained quadrature Gaussian channel and show that the optimal input distribution is concentrated to a finite number of uniform phase circles within the amplitude constraint. Recently, Raginsky [15] proved using relations connecting information and estimation theories that if the amplitude constraint is less than 1.05 in a unit noise power AWGN channel, then the capacity achieving input distribution is binary with two equal mass points that are symmetric around the origin.

\section{SySTEM MODEL}

We consider the scalar AWGN channel characterized by the input $X$, output $Y$, additive noise $N$ with unit normal distribution $\mathcal{N}(0,1)$, and a battery (see Fig. 1). Input and output alphabets are taken as real numbers. Energy enters the system from a power source that supplies $E_{i}$ units of energy in the $i$ th channel use where $E_{i} \geq 0 . E_{1}, \ldots, E_{n}$ is the time sequence of supplied energy in $n$ channel uses. $E_{i}$ is an i.i.d. sequence with average value $P$, i.e., $E\left[E_{i}\right]=P$, for all $i$.

$E_{\max }$ units of energy can be stored in the battery and the existing energy in the battery can be retrieved without any loss. For convenience, we assume that the energy stored and depleted from the battery are for only communication purposes (e.g., we do not consider the energy required for processing). Moreover, our focus here is on the case where $E_{\max }=\infty$, and hence, energy overflow does not occur and incoming energy can always be saved in the battery. This assumption is especially valid for the current technology in which batteries have very large energy storage capacities compared to the rate of harvested energy flow: $E_{\text {max }} \gg P .{ }^{1}$ The battery is initially empty and energy needed for communication of a message is obtained from the arriving energy during the transmission of the corresponding codeword subject to causality. In particular, $E_{i}$ units of energy is added to the battery and $X_{i}^{2}$ units of energy is depleted from the battery in the $i$ th channel use. This is illustrated in Fig. 2.

This brings us to the following cumulative power constraints on the channel inputs based on the causality of energy usage:

$$
\sum_{i=1}^{k} X_{i}^{2} \leq \sum_{i=1}^{k} E_{i}, \quad k=1, \ldots, n .
$$

${ }^{1}$ We have studied the other extreme where $E_{\max }=0$ in [16]. We leave the finite $E_{\max }$ case, i.e., $0<E_{\max }<\infty$, for future research. 


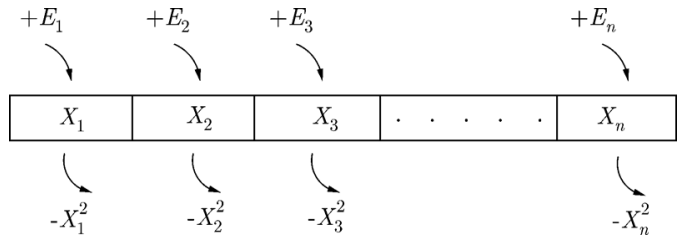

Fig. 2. $\quad E_{i}$ is the energy arriving and $X_{i}^{2}$ is the energy used at time $i$ where $X_{i}$ is the $i$ th code symbol.

Note that the constraints in (1) are upon the support set of the random variables $X_{i}$. The first constraint restricts the support set of $X_{1}$ to $\left[-\sqrt{E_{1}}, \sqrt{E_{1}}\right]$. The second constraint is $X_{1}^{2}+X_{2}^{2} \leq$ $E_{1}+E_{2}$. In general, letting $S_{i}$ denote $\left[\sum_{j=1}^{i-1}\left(E_{j}-X_{j}^{2}\right)\right]^{+}$, in channel use $i$, the symbol $X_{i}$ is subject to the constraint $X_{i}^{2} \leq$ $E_{i}+S_{i}$.

The input constraints in (1) introduce memory (in time) in the channel inputs. Randomness in $E_{i}$ makes the problem similar to fading channels in that the state of recharge process (i.e., low or high $E_{i}$ ) affects instantaneous quality of communication. Moreover, this time variation in the recharge process allows opportunistic control of transmit energy as in fading channels. However, recharged energy can be saved in the battery for future use unlike a fading state. In fact, we will see that this nature of energy arrivals renders saving energy in the battery more advantageous for later use when a peak occurs in the recharge process, as opposed to opportunistically riding the peaks.

Codebook $\mathcal{C}^{n}=\left(n, 2^{n R_{n}}, \epsilon_{n}\right)$ is defined by the code length $n$, the code size $2^{n R_{n}}$, and the probability of error $\epsilon_{n}$. The messages in the set $\left\{1, \ldots, 2^{n R_{n}}\right\}$ are equally likely. Encoding function is $f_{k}^{n}:\left\{1, \ldots, 2^{n R_{n}}\right\} \rightarrow \mathcal{X}, k=1, \ldots, n$ and the decoding function is $\phi^{n}: \mathcal{Y}^{n} \rightarrow\left\{1, \ldots, 2^{n R_{n}}\right\}$. Here, encoding and decoding are performed independent of energy information. In fact, energy information at the encoder/decoder does not improve the capacity as will be apparent in the following sections. There are two separate causes of error. The first one is that a codeword does not satisfy the input constraints at a particular channel use. In this case, the transmitter experiences an energy shortage to transmit the codeword and this event is counted as an error event. The second cause of error is the decoding error at the receiver. If the received signal is decoded to a message that is different from the message sent, then an error occurs. Accordingly, the error event is defined as the union of two events: $\varepsilon^{n}=\varepsilon_{1}^{n} \cup \varepsilon_{2}^{n}$ where $\varepsilon_{1}^{n}$ is the energy shortage event, and $\varepsilon_{2}^{n}$ is the decoding error event. The overall probability of error is $\epsilon_{n}=\operatorname{Pr}\left(\varepsilon^{n}\right)$.

\section{CAPACITY}

We will invoke the general capacity formula of Verdu and Han [17]. For fixed $n$, let $F^{n}$ be the joint cumulative distribution function of the random variables $\left\{X_{i}\right\}_{i=1}^{n}$ and let $\mathcal{F}^{n}$ be the set of $n$ variable joint cumulative distribution functions that satisfy the constraints in (1). Since the AWGN channel is an information-stable channel [17], the capacity of the channel in Fig. 1 with constraints in (1) is

$$
C=\lim _{n \rightarrow \infty} \max _{F^{n} \in \mathcal{F}^{n}} \frac{1}{n} I\left(X^{n} ; Y^{n}\right) .
$$

In general, for an AWGN channel, the capacity achieving input distribution is in the form of a product of marginal distributions (independent distribution) [17]. However, note that the power constraints in our problem create dependence among the random variables. The constraint on $X_{i+1}$ is dependent on the given values of $X_{j}, j \leq i$. Though in a classical AWGN channel independent processes achieve higher mutual information than the ones with the same marginal distribution but with correlation [17], the capacity that we seek in this problem does not let the process be independent. This problem falls in the family of problems of finding capacity under dependence constraints on code symbols which is by itself interesting and less studied.

An upper bound for $C$ is the corresponding AWGN capacity with average power constrained to average recharge rate $P$, as $\frac{1}{n} \sum_{i=1}^{n} X_{i}^{2} \leq \frac{1}{n} \sum_{i=1}^{n} E_{i}$ and by the i.i.d. nature of $E_{i}$, invoking the strong law of large numbers [18], $\frac{1}{n} \sum_{i=1}^{n} E_{i} \rightarrow P$ with probability one. Therefore, each codeword satisfying the constraints in (1) automatically satisfies $\lim _{n \rightarrow \infty} \frac{1}{n} \sum_{i=1}^{n} X_{i}^{2} \leq P$ with probability one. However, the reverse is not true. If a codeword satisfies the average power constraint, it does not necessarily satisfy the constraints in (1). Hence, the channel capacity under the energy constraints in (1) is bounded by the following for almost all realizations of the energy arrival process:

$$
C \leq \frac{1}{2} \log (1+P) .
$$

The main result of this paper is that the upper bound in (3) can be achieved, as stated in the following theorem.

Theorem 1: The capacity of an AWGN channel with channel inputs constrained by the random i.i.d. energy arrival sequence $\left\{E_{i}\right\}_{i=1}^{\infty}, E\left[E_{i}\right]=P$ and an infinite-sized battery is equal to the classical AWGN capacity with average power constraint $P$

$$
C=\frac{1}{2} \log (1+P) \text {. }
$$

In the next two sections, we develop two different achievability schemes that achieve the capacity given in Theorem 1 .

\section{SAVE-And-TRAnSmit Scheme}

While designing the codebook and the encoding/decoding rule, a first approach could be to optimize the codebook design subject to the input constraints in (1) so that the occurrence of the error event $\varepsilon_{1}^{n}$ is eliminated from the beginning. Instead, we propose a scheme that implements a save-and-transmit principle which averages out the randomness in energy arrivals first, and then performs channel coding to counter errors due to the randomness in the channel.

In the save-and-transmit scheme, data transmission is performed in two phases: first the saving phase where the battery is fueled with energy and then the transmission phase where information carrying code symbols are sent. Therefore, we will consider the sequence of codes with code length $n$ such that the first $h(n) \in o(n)$ symbols of each codeword are zero and the remaining $n-h(n)$ code symbols are the information carrying symbols, where $o(n)$ denotes the class of functions that scale slower than $n$. We particularly consider $h(n) \in o(n)$ such that 


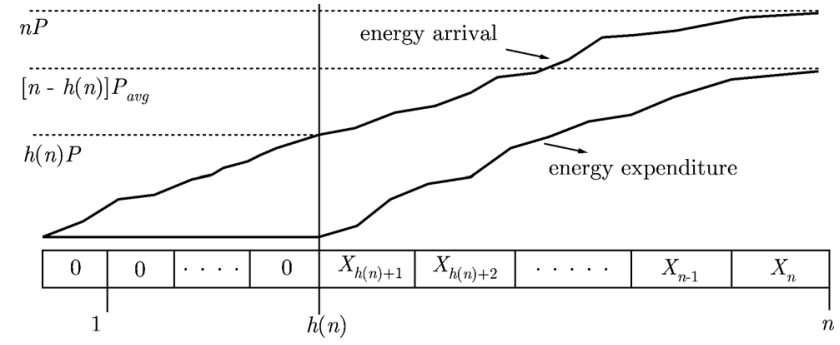

Fig. 3. Save-and-transmit scheme: first, $h(n)$ code symbols are identically zero for all codewords, and the remaining $n-h(n)$ code symbols are selected as i.i.d. Gaussian distributed with variance $P_{\text {avg }}$. Error of the first type occurs if the energy arrival curve crosses the energy expenditure curve.

$h(n) \rightarrow \infty$ and $n-h(n) \rightarrow \infty$ as $n \rightarrow \infty$. The reason for considering $o(n)$ functions for the saving period is to allow sufficient number of channel uses for the data transmission period so that no loss is incurred in achievable rates.

In the first $h(n)$ symbols, no energy is spent for communication and battery energy is increased. In the remaining $n-h(n)$ channel uses, information carrying symbols which are chosen as independent random variables from the (capacity achieving) Gaussian distribution with mean zero and variance $P_{\text {avg }}$ is transmitted. That is, for $k=1, \ldots, h(n)$, we have $f_{k}^{n}(m)=0$ for all $m \in\left\{1, \ldots, 2^{n R_{n}}\right\}$. For $k=h(n)+1, \ldots, n, f_{k}^{n}(m)$ is selected as independent samples of a zero mean and variance $P_{\text {avg }}$ Gaussian random variable for all $m \in\left\{1,2, \ldots, 2^{n R_{n}}\right\}$.

We note that the save-and-transmit scheme does not use any information of the recharge process $\left\{E_{i}\right\}_{i=1}^{\infty}$. Irrespective of the realization of $\left\{E_{i}\right\}_{i=1}^{\infty}$, we introduce $h(n) \in o(n)$ amount of delay to save energy and then transmit with average power $P_{\text {avg }}<P$. We aim to prove that there exists $h(n) \in o(n)$ that can guarantee sufficient energy savings to prevent any energy shortages in the transmission phase, which, in turn, implies that the energy shortage probability $\varepsilon_{1}^{(n)}$ and probability of decoding error $\varepsilon_{2}^{(n)}$ both go to zero and rates arbitrarily close to the upper bound in (3) are achieved.

For $h(n) \rightarrow \infty$, by the strong law of large numbers, at time index $h(n)$, about $h(n) P$ amount of energy is saved in the battery with high probability. We argue that if $P_{\text {avg }}<P$, in the remaining $n-h(n)$ channel uses, this saved energy together with energy entering the system is sufficient to provide the energy needed for data transmission (see Fig. 3). We will formalize this argument in the following lemma.

Lemma 1: Assume $h(n) \in o(n)$ with $\lim _{n \rightarrow \infty} h(n)=\infty$. The save-and-transmit scheme satisfies the input constraints in (1) with probability arbitrarily close to one provided that $P_{\text {avg }}<$ $P$.

A proof of Lemma 1 is provided in Appendix A. Lemma 1 says that in the save-and-transmit scheme if $P_{\text {avg }}<P$, a saving period $h(n) \in o(n)$ with $h(n) \rightarrow \infty$ is sufficient to collect an initial amount of energy to prevent energy shortages during the transmission phase. The proof requires an application of the strong law of large numbers along with the tail behavior of sums of i.i.d. random variables. For instance, we can select $h(n)=$ $\log (n)$ as $\log (n) \in o(n)$ and $\log (n) \rightarrow \infty$ and by Lemma 1 , it is guaranteed that the probability of any energy shortages goes to zero. The achievable rate for this scheme with decoding error approaching zero is [17]

$$
\begin{aligned}
\lim _{n \rightarrow \infty} \frac{1}{n} I\left(\mathbf{X}^{n} ; \mathbf{Y}^{n}\right) & =\lim _{n \rightarrow \infty} \frac{1}{n} \sum_{j=h(n)+1}^{n} I\left(X_{j} ; Y_{j}\right) \\
& =\lim _{n \rightarrow \infty} \frac{n-h(n)}{2 n} \log \left(1+P_{\text {avg }}\right) \\
& =\frac{1}{2} \log \left(1+P_{\text {avg }}\right) .
\end{aligned}
$$

Since $\log ($.$) is continuous, R<\frac{1}{2} \log (1+P)$ can be achieved by choosing $P_{\text {avg }}=P-\epsilon$, and therefore, the capacity in Theorem 1 is achievable by the save-and-transmit scheme.

Using the advantage of having a battery to buffer energy, the save-and-transmit scheme first eliminates the uncertainty in the energy arrivals, and then copes with the uncertainty in the channel by means of appropriate channel coding. The actual data transmission starts with an $o(n)$ delay and the capacity with average power constrained to the average recharge rate can be achieved.

\section{A. Case of $P_{\mathrm{avg}}=P$}

We have seen that the save-and-transmit scheme can achieve rates arbitrarily close to the capacity by saving energy in the first $h(n) \in o(n)$ channel uses and then transmitting with zero-mean Gaussian distributed codewords of power $P-\epsilon$. Although this scheme proves the desired capacity result, in this section we consider the case of $P_{\text {avg }}=P$. This is a technically challenging case where the average energy entering the battery is exactly the same as the average energy exiting the battery. We will establish that the capacity could be achieved with the save-and-transmit scheme even when $P_{\text {avg }}=P$. However, we have to modify the scheme and the assumptions on the statistics of energy arrivals. In particular, in this case, the saving period $h(n)=\log (n)$ is not sufficient to guarantee that the energy constraints are satisfied with probability one. That is, the hypothesis in Lemma 1 should be refined for this particular case. To see this, assume that $P_{\text {avg }}=P$ and consider $h(n)=\sqrt{n}$. Note that $\sqrt{n}=\omega(\log (n))$ where $\omega(\log (n))$ denotes the class of functions $f$ such that for all $k \geq 0$, there exists sufficiently large $n$ that satisfies $f(n)>k \log (n)$. In this case, as shown in Appendix B, we have

$$
\lim _{n \rightarrow \infty} \operatorname{Pr}\left(\sum_{i=1}^{n} X_{i}^{2}>\sum_{i=1}^{n} E_{i}\right)=\Phi\left(\frac{P}{a}\right)
$$

where, assuming that $E_{i}$ has a finite-variance $\sigma_{E}^{2}, a$ is given as $a^{2} \triangleq \sigma_{E}^{2}+2 P^{2}$ (see Appendix B), and $\Phi(x)$ is the cumulative distribution function of a unit normal random variable, i.e., $\Phi(x)=\int_{x}^{\infty} \frac{1}{\sqrt{2 \pi}} e^{-\frac{\tau^{2}}{2}} d \tau$. Hence, if $h(n)=\sqrt{n}$, energy shortages occur with a nonzero probability. In fact, with $h(n)=\log (n)$, as also shown in Appendix B

$$
\lim _{n \rightarrow \infty} \operatorname{Pr}\left(\sum_{i=1}^{n} X_{i}^{2}>\sum_{i=1}^{n} E_{i}\right)=\Phi(0)=\frac{1}{2}
$$

and hence energy shortages occur with probability higher than $\frac{1}{2}$ when $h(n)=\log (n)$. After these pessimistic results, it is of 
question whether we can find $h(n) \in o(n)$ that guarantees that no energy shortages occur in the $P_{\text {avg }}=P$ case. Clearly, such $h(n)$ must scale at least as fast as $\sqrt{n}$.

We are able to find a family of $h(n) \in o(n)$ under some mild regulatory assumptions on the probability distribution of the energy arrivals, as stated in the following lemma.

Lemma 2: Suppose that $E_{i}$, the energy arrival random variable, satisfies $E\left[e^{E_{i}^{\gamma}}\right]<\infty$ for some $0<\gamma<1$. Then, the save-and-transmit scheme satisfies the constraints in (1) with probability arbitrarily close to one for $h(n)=n^{\frac{1}{\alpha}}(\log (n))^{\frac{1}{\gamma}}$, where $1<\alpha \leq 2$.

A proof of Lemma 2 is provided in Appendix C. It is based on a recent strong law for sums of i.i.d. random variables that is originally proved in [19] and the fact that $E\left[e^{\left|X_{i}\right|^{2 \gamma}}\right]<\infty$ for the Gaussian distributed $X_{i}$ in the assumed range of $\gamma$. Lemma 2 says that under mild conditions on the energy harvesting process $E_{i}$, there exists $h(n)$ that scales faster than $\sqrt{n} \log (n)$ such that we can save sufficient amount of initial energy in the saving phase to guarantee that there will be no energy shortages during the transmission phase even when the average energy exiting the system $P_{\text {avg }}$ (codebook power) exactly equals the average energy entering the system $P$ (recharge rate). For example, if $E\left[e^{\sqrt{E_{i}}}\right]<\infty$ is satisfied, then $h(n)=\sqrt{n}(\log (n))^{2}$ guarantees no energy shortages during the transmission. We note that $E\left[e^{\sqrt{E_{i}}}\right]<\infty$ is true for a large class of random variables including bounded support, exponential, and $\chi^{2}$ distributed random variables. Since $h(n)=n^{\frac{1}{\alpha}}(\log (n))^{\frac{1}{\gamma}} \in o(n)$ for $1<\alpha \leq 2$ and $0<\gamma<1$, the saving period does not result in any loss in the achievable rate, and thus, the save-and-transmit scheme achieves the capacity for the case of $P_{\text {avg }}=P$.

\section{Best-EfFort-Transmit Scheme}

The input constraints in (1) impose that the codewords must satisfy the energy constraint in every channel use. However, it is possible to achieve a reliable communication rate even if code symbols satisfy the energy constraints in almost every channel use except possibly a finite number of them. Therefore, transmission of data in two phases may not be necessary. In this section, we propose an alternative single-phase scheme that attains the capacity using Gaussian codewords subject to the availability of energy in the battery. We call this new scheme the best-effort-transmit scheme.

Let $X^{n}=\left(X_{1}, X_{2}, \ldots, X_{n}\right)$ be a codeword of length $n$ where $X_{i}$ is the code symbol to be transmitted in channel use $i$ and the codebook be $\mathcal{C}^{n}$. The codebook that the two parties agree upon is determined by generating independent Gaussian distributed random samples with mean zero and variance $P_{\text {avg }}$, i.e., $\mathcal{C}^{n}$ is a randomly generated codebook. Let $S(i)$ be the battery energy just before the $i$ th channel use starts. In the best-effort-transmit scheme, the code symbol $X_{i}$ can be put to the channel if $S(i) \geq X_{i}^{2}$. Otherwise, the transmitter puts a code symbol 0 to the channel as battery does not have sufficient energy to transmit symbol $X_{i}$. Hence, the battery energy is updated according to the following rule:

$$
S(i+1)=S(i)+E_{i}-X_{i}^{2} \mathbf{1}\left(S(i) \geq X_{i}^{2}\right) .
$$

The energy updates in (10) are analogous to the queue updates in classical slotted systems [3]. Unlike in classical queuing in data networks, the energy queue is desired to be unstable so that there is always sufficient energy to transmit code symbols.

We say that the symbol $X_{i}$ is infeasible if there is not sufficient energy to send $X_{i}$, i.e., $S(i)<X_{i}^{2}$. Note that the codewords in the best-effort-transmit scheme are allowed to violate the energy constraints in (1); however, the actual channel inputs always satisfy the energy feasibility constraints in (1) in all channel uses. Therefore, there is no error due to energy shortages in the codewords and we only account for the decoding error at the receiver in the best-effort-transmit scheme. The input to the channel is $X_{i} \mathbf{1}\left(S(i) \geq X_{i}^{2}\right)$. Consequently, the codeword in the codebook may be different from what is actually transmitted. That is, in the transmitted codeword, some of the symbols in the actual codeword in the codebook are replaced with zeros. This causes a mismatch between the encoder and the decoder. Occurrences of such mismatches are determined by the dynamics of the available energy in the battery, which, in turn, is determined by the energy arrival and channel input processes. We are able to show that the resulting mismatch is negligible and communication with rates arbitrarily close to $\frac{1}{2} \log (1+P)$ is possible. We start with the following key observation.

Lemma 3: In the best-effort-transmit scheme, if $P_{\text {avg }}<P$, for almost all realizations of the energy arrival process $\left\{E_{i}\right\}_{i=1}^{n}$ and the codebook $\mathcal{C}^{n}$, the code symbols are infeasible only at finitely many channel uses as $n$ grows to infinity.

Proof: Let $P_{\text {avg }}=P-\epsilon$ and note that $E_{i}-X_{i}^{2}-\epsilon$ is a zero-mean sequence. By the strong law of large numbers, only finitely many of the sequence of events $\left\{\frac{1}{n} \sum_{i=1}^{n}\left(E_{i}-X_{i}^{2}-\epsilon\right)<-\delta\right\}_{n=1}^{\infty}$ occur for any $\delta>0$. Selecting $\delta=\epsilon$, this is equivalent to the assertion that for only finitely many of the indices $\sum_{i=1}^{n}\left(E_{i}-X_{i}^{2}\right)<0$. Note that $\sum_{i=1}^{n}\left(E_{i}-X_{i}^{2} 1\left(S(i) \geq X_{i}^{2}\right)\right) \geq \sum_{i=1}^{n}\left(E_{i}-X_{i}^{2}\right)$. This implies that $\sum_{i=1}^{n}\left(E_{i}-X_{i}^{2} \mathbf{1}\left(S(i) \geq X_{i}^{2}\right)\right)<0$ occurs for only finitely many of the indices. Therefore, code symbols are infeasible, i.e., there is a shortage of energy in the battery, only in finitely many channel uses.

In an AWGN channel, with a codebook generated with i.i.d. Gaussian samples with variance $P_{\text {avg }}$, rates arbitrarily close to $\frac{1}{2} \log \left(1+P_{\mathrm{avg}}\right)$ can be achieved with probability of decoding error approaching zero [20]. The achievability is based on random coding and joint typical decoding, which checks whether the received vector is jointly typical with a codeword from the codebook, and the associated joint asymptotic equipartition property (AEP). In the best-effort-transmit scheme, there are mismatches between the codewords in the codebook and the actual transmitted codewords. However, if the average power of the codewords $P_{\text {avg }}$ is smaller than the average recharge rate, $E\left[E_{i}\right]=P$, in view of Lemma 3 , such mismatches are only finitely many, as the number of channel uses goes to infinity. This enables us to use joint typicality decoding at the receiver to reliably decode the message. This is true essentially because the joint AEP (see [20, Th. 7.6.1,]) is based on laws of large numbers which are unaffected by finite number of alterations, as the number of samples goes to infinity. More specifically, we prove that in the best-effort-transmit scheme, if $P_{\text {avg }}<P$ and 
joint typicality decoder is used at the receiver, the probability of decoding error goes to zero as the block length $n$ gets large. The following lemma provides the desired step to prove this result.

Lemma 4: Let $x^{n}$ be an arbitrary codeword in the codebook $\mathcal{C}^{n}$ and $y^{n}$ be the corresponding received signal in the best-effort-transmit scheme. Let $A_{\epsilon}^{n}$ denote the set of jointly typical sequence tuples with respect to $p(x, y)=p(x) p(y \mid x)$ where $p(x)=\mathcal{N}\left(0, P_{\text {avg }}\right)$ and $p(y \mid x)=\mathcal{N}(x, 1)$. Assume $P_{\text {avg }}<P$. The following statements hold for almost all realizations of the energy arrival process $\left\{E_{i}\right\}_{i=1}^{n}$.

1) $\operatorname{Pr}\left(\left(x^{n}, y^{n}\right) \in A_{\epsilon}^{n} \mid E_{1}, \ldots, E_{n}\right) \rightarrow 1$ as $n \rightarrow \infty$.

2) Let $\tilde{x}^{n} \neq x^{n}$ be another codeword in $\mathcal{C}^{n}$. For sufficiently large $n$, we have

$$
\operatorname{Pr}\left(\left(\tilde{x}^{n}, y^{n}\right) \in A_{\epsilon}^{n} \mid E_{1}, \ldots, E_{n}\right) \leq 2^{-n(I(X ; Y)-3 \epsilon)}
$$

where $I(X ; Y)$ is the mutual information between single-letter random variables $X$ and $Y$ jointly distributed as $p(x, y)$.

Proof of Lemma 4 directly follows from Lemma 3 and [20, Th. 7.6.1,]. In particular, whenever $P_{\text {avg }}<P$, for almost all realizations of the energy arrival process $\left\{E_{i}\right\}_{i=1}^{\infty}$ and codewords in the codebook, there exists a finite number $N>0$ such that none of the code symbols with index $i>N$ in any codeword is altered due to the insufficiency of the battery energy. Therefore, given $E_{1}, \ldots, E_{n}$ and codeword $x^{n}$ with $n \gg N$, the last $n-N$ received symbols of $y^{n}$ are the channel responses to the last $n-N$ code symbols of $x^{n}$. As $n$ gets large, the effect of the first $N$ received symbols becomes negligible, and hence, the received signal is jointly typical with the transmitted codeword for all $\epsilon>0$ and sufficiently large $n$. In view of [20, Th. 7.6.1,], Lemma 4 holds.

We note that the message, channel noise sequence, and energy arrival sequence are mutually independent. Consequently, we combine Lemma 4, [20, Th. 7.6.1,], and the achievability part of [20, Th. 7.7.1,] and conclude that if $P_{\text {avg }}<P$ and the receiver uses a joint typicality decoder, then in the best-effort-transmit scheme the probability of decoding error vanishes as $n$ grows to infinity for almost all realizations of the energy arrival process. Finally, as the probability of decoding error approaches zero in a randomly designed codebook $\mathcal{C}^{n}$, there must exist a codebook such that the probability of decoding error approaches zero for almost all realizations of the energy arrival process. Since rate $R=\frac{1}{2} \log \left(1+P_{\text {avg }}\right)$ is achievable under an average power constraint $P_{\text {avg }}<P$, we have the desired result.

Theorem 2: In the best-effort transmit scheme, rates arbitrarily close to $\frac{1}{2} \log (1+P)$ are achievable.

\section{DISCUSSION}

We now comment on the two capacity achieving schemes. In the save-and-transmit scheme, the available channel uses are divided into two phases. The saving phase duration $h(n)$ is selected as $o(n)$ with $\lim _{n \rightarrow \infty} h(n)=\infty$ and this, along with the unlimited sized battery, allows averaging out the uncertainty in the available energy. Remaining $n-h(n)$ channel uses are used for channel coding with an average power constraint equal to the average recharge rate. Although for a fixed block length $n$, there is a nonzero probability that available energy in the battery is not sufficient to put the designed code symbol into the channel, this probability approaches zero as $n$ gets large. The save-and-transmit scheme does not use the information about the amount of available energy in the battery at any given time. In contrast, the best-effort-transmit scheme uses this information. Whenever battery energy is sufficient to send the designed code symbol, that code symbol is put to the channel, and if the energy in the battery falls short of sending the designed code symbol, a zero symbol is put to the channel. The best-effort-transmit scheme interacts with the battery energy level and adapts the transmission so that the message is reliably transmitted. It is clear that the extra information that the best-effort-transmit scheme uses brings no advantage in terms of the achievable rates. However, this information enables the transmitter start transmission of the message right away and codewords are infeasible in at most finitely many channel uses. In the save-and-transmit scheme, the saving period $h(n)$ has to grow to infinity for eradicating any energy shortages throughout the data transmission, which is a consequence of the lack of interaction between the channel input and the battery energy level.

We note that both save-and-transmit and best-effort-transmit schemes need unlimited sized batteries. It is more obvious that the save-and-transmit scheme needs an unlimited sized battery, since the battery energy needs to go to infinity in the saving phase as the block length gets large. The fact that the best-effort-transmit scheme also needs an unlimited sized battery is less obvious. While the best-effort-transmit scheme starts transmission right away, since $P_{\text {avg }}<P$, eventually, the battery energy goes to infinity. In fact, this is the reason that energy shortages occur only in finitely many channel uses. Essentially, after a large enough channel use index, the battery has so much energy that no energy shortages occur.

It is also worth noting that stochastic energy levels at the transmitter connect the problem considered here to the problem of communicating over state-dependent channels with state information available at the transmitter only, where the state is the energy arrival sequence [16]. Although the availability of the state information at the transmitter and/or receiver boosts the capacity of state-dependent channels in general [16], the capacity of the AWGN channel with an energy harvesting transmitter with an unlimited battery does not change whether the energy arrival information is available at the transmitter/receiver or not. In fact, in the save-and-transmit and best-effort-transmit schemes, neither the transmitter nor the receiver needs to know the energy arrival information.

Moreover, we note that memory may affect the capacity of state-dependent channels in general [21]; however, the capacity of the AWGN channel with an energy harvesting transmitter with an unlimited battery is invariant to the memory in the energy arrival process, so long as the energy arrival process is stationary and ergodic. That is, an i.i.d. energy arrival process and a non-i.i.d. energy arrival process with the same average arrival rate will yield the same capacity so long as the non-i.i.d. energy arrival process is stationary and ergodic. Clearly, the converse argument in (3) is still valid in this case since the 
sample mean of the energy arrival process has the same limiting property. Furthermore, the save-and-transmit and best-effort-transmit schemes achieve the capacity in this case on the grounds that laws of large numbers hold for stationary and ergodic class of random processes [18]. In particular, Lemmas 1, 3 , and 4 generalize to this class of energy arrival processes after simple modifications in their proofs.

Finally, we remark that the save-and-transmit and best-effort-transmit schemes and the capacity results presented in this paper can be straightforwardly generalized to a single-user fading or multiple-input multiple-output channel, or various multiuser channel models, such as the multiple access channel, broadcast channel, interference channel, relay channel, and wiretap channel, etc., with energy harvesting transmitters, as long as the transmitters are equipped with unlimited sized batteries. With save-and-transmit and best-effort-transmit schemes, we can achieve the rates that are achievable with corresponding average power constraints.

\section{Optimal Power Management in a Large Time Scale}

We have seen that energy harvesting systems can achieve classical AWGN capacity if the recharge process is i.i.d. and the block length is sufficiently large. However, the recharge process can deviate from its i.i.d. characteristic in a large time scale. In particular, the mean value of the recharge process may vary after a long duration that is sufficient to decode the transmitted message. In the classical example of sensor nodes fueled with solar power, mean recharge rate changes depending on the time of the day. As an example, the mean recharge rate may vary in 1-h frames and the sensor may be on for $12 \mathrm{~h}$ a day, in which case, a careful management of energy expenditure in each frame will be required to optimize the average performance during the day. Consider $L$ time frames (see Fig. 4). The duration of each frame is $T_{s}$. For each frame $i=1, \ldots, L$, the average recharge rate is $P_{i}$ and $Q_{i}$ units of power is allocated for data transmission. Hence, in frame $i, P_{i} T_{s}$ units of energy enters the system and $Q_{i} T_{s}$ units of energy is spent. $T_{s}$ is sufficiently large so that $T_{s} \frac{1}{2} \log \left(1+Q_{i}\right)$ bits are reliably sent in this duration. Assuming zero initial energy in the battery and unlimited battery storage capacity, the causality constraint on the energy expenditure due to the energy arrivals is

$$
\sum_{i=1}^{\ell} Q_{i} \leq \sum_{i=1}^{\ell} P_{i}, \quad \ell=1, \ldots, L .
$$

The designer knows the mean recharge rates $P_{i}$ for all $i$ and calculates $Q_{i}$ before the communication and adjusts the average power of codewords in frame $i$ to $Q_{i}$ during transmission. ${ }^{2} \mathrm{We}$ allocate a transmit power to each frame subject to causality constraint so that average throughput in $L$ frames is maximized

$$
\begin{array}{ll}
\max & \frac{1}{L} \sum_{i=1}^{L} \frac{1}{2} \log \left(1+Q_{i}\right) \\
\text { s.t. } & \sum_{i=1}^{\ell} Q_{i} \leq \sum_{i=1}^{\ell} P_{i}, \quad \ell=1, \ldots, L .
\end{array}
$$

${ }^{2}$ Changing the average power of codewords requires using different codebooks in each frame. However, scaling a common codebook by frame power $Q_{i}$ works as well. This can also be interpreted as a codebook with dynamic power allocation [21] in slow time variation.

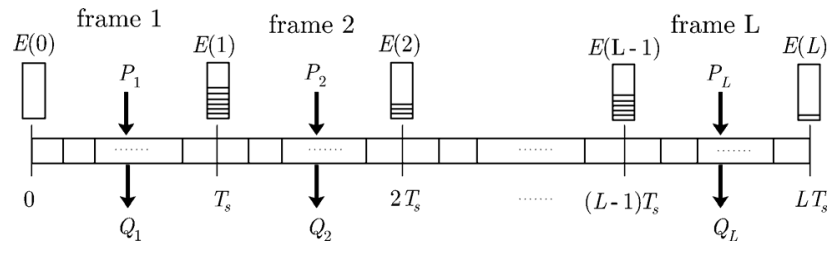

Fig. 4. $L$ large time frames. Each frame is sufficiently long to achieve AWGN capacity with average power constrained to the allocated power in that frame.

A first observation about the optimization problem in (13) is that any power vector with $\sum_{i=1}^{L} Q_{i}<\sum_{i=1}^{L} P_{i}$ is strictly suboptimal because $\log ($.$) is a monotone increasing function. Hence,$ the constraint in (13) for $\ell=L$ can be cast as an equality. We will refer to power vectors satisfying (13) as feasible in the following. We denote the solution of the optimization problem in (13) as $\mathbf{Q}^{*}=\left[Q_{1}^{*}, \ldots, Q_{L}^{*}\right]$. The rest of this section is devoted to characterizing the optimal power vector $\mathbf{Q}^{*}$.

We will solve the optimization problem in (13) by using tools from majorization theory and Schur convexity [22]. We start with the following definition.

Definition 1: Let $\mathbf{x}=\left[x_{1}, \ldots, x_{n}\right]$ and $\mathbf{y}=\left[y_{1}, \ldots, y_{n}\right]$ be two $n$-dimensional nonnegative vectors and let $x_{(j)}$ denote the $j$ th largest component of $\mathbf{x}$. Then, $\mathbf{x}$ is said to be majorized by $\mathbf{y}$, denoted by $\mathbf{x} \preceq \mathbf{y}$, if

$$
\begin{aligned}
\sum_{j=1}^{k} x_{(j)} & \leq \sum_{j=1}^{k} y_{(j)}, \quad k=1, \ldots, n-1 \\
\sum_{j=1}^{n} x_{j} & =\sum_{j=1}^{n} y_{j} .
\end{aligned}
$$

The majorization relation measures how spread a vector is from its mean value. It can be shown that [22] any $n$-dimensional vector $\left[x_{1}, \ldots, x_{n}\right]$ majorizes the constant $n$-dimensional vector with each coordinate equal to $\frac{\sum_{i} x_{i}}{n}$. If a function $f\left(x_{1}, \ldots, x_{n}\right)$ is Schur convex, then $\left[x_{1}, \ldots, x_{n}\right] \preceq$ $\left[y_{1}, \ldots, y_{n}\right]$ implies $f\left(x_{1}, \ldots, x_{n}\right) \leq f\left(y_{1}, \ldots, y_{n}\right)$. If $-f$ is Schur convex, then $f$ is Schur concave. The following result [22] will be useful.

Lemma 5: If $f\left(x_{1}, \ldots, x_{n}\right)=\sum_{i=1}^{L} g\left(x_{i}\right)$ where $g(x)$ is convex, then $f$ is Schur convex.

The objective function in (13) is Schur concave since $\log ($. is concave. Therefore, the solution of the optimization problem in (13) is the transmit power vector $\mathbf{Q}^{*}$ which is the most majorized feasible power vector, i.e., $\mathbf{Q}^{*}$ is the optimal transmit power vector if $\mathbf{Q}^{*} \preceq \mathbf{Q}$ for all feasible $\mathbf{Q}$. Thus, we need to find the most majorized feasible transmit power vector.

In order to understand how the most majorized feasible power vector may look like, we first consider the simplest scenario. Suppose that $c L T_{s}$ amount of energy is available in the battery for some nonnegative constant $c$ and the recharge process is zero. In this case, the uniform power vector $Q_{i}=c$ is majorized by every other feasible vector. For the original problem, if the constant vector $Q_{i}=\frac{\sum_{j=1}^{L} P_{j}}{L}$ is feasible, then it is majorized by any other feasible vector. However, the constant vector may not be in the feasible set. This is due to the causality of energy 
arrivals: while energy can be spread to future to equalize powers as much as possible, if large amounts of energy arrive in later frames they cannot be spread to earlier frames to equalize the powers.

We now generalize the intuition obtained from the previous discussion for an arbitrary energy arrival case. In particular, we adapt the idea of allocating power as constant as possible for the general case taking the causality constraints into consideration using an energy curve approach. This approach has appeared in the context of energy minimal transmission in [23] where authors characterize energy minimal policy in a delay-limited scenario as the tightest line below the data arrival curve. Later in the context of energy harvesting systems, similar structural properties have been observed in the policies with minimum transmission completion time in [6] and [7]. In this paper, we will obtain the optimal power management vector as the tightest line below the cumulative energy arrivals. First, we define the cumulative energy arrivals as

$$
e(i)=\sum_{j=1}^{i} P_{j}, \quad i=1, \ldots, L
$$

and by convention $e(0)=0$. Since the power vector should be made as constant as possible, it is determined such that its cumulative energy expenditure is the tightest piecewise linear curve below $e(i)$. Therefore, the algorithm divides the frames into $K$ constant power bands $L_{k}+1 \leq i \leq L_{k+1}, k=$ $1, \ldots, K$. In particular, the optimal power vector $Q_{i}^{*}$ is constant over $L_{k}+1 \leq i \leq L_{k+1}, k=1, \ldots, K$. By convention, $L_{1}=0, L_{K+1}=L$, and the remaining $L_{k}$ are determined as follows:

$$
L_{k}=\arg \min _{i \in\left\{L_{k-1}+1, \ldots, L\right\}} \frac{e(i)-e\left(L_{k-1}\right)}{i-L_{k-1}} .
$$

As we find the tightest line below the energy arrival curve, $Q_{i}^{*}$ takes the constant value $\frac{e\left(L_{k+1}\right)-e\left(L_{k}\right)}{L_{k+1}-L_{k}}$ over the $k$ th band $L_{k}+$ $1 \leq i \leq L_{k+1}$. We claim that the following power vector is optimal:

$$
Q_{i}^{*}=\frac{e\left(L_{k}\right)-e\left(L_{k-1}\right)}{L_{k}-L_{k-1}}, \quad i=L_{k-1}+1, \ldots, L_{k} .
$$

To prove optimality, we show in the next theorem that $Q_{i}^{*}$ obtained via this procedure is the most majorized feasible transmit power vector.

Theorem 3: $\mathbf{Q}^{*}$ defined through (17) and (18) is the most majorized feasible power vector.

A proof of the Theorem 3 is provided in Appendix D which uses direct verification of the majorization conditions in (14) and (15). Since the objective function is Schur concave, by Theorem $3, \mathbf{Q}^{*}$ in (18) is the optimal power vector.

An illustration of the operation of the optimum power management algorithm is presented for a four frame case in Fig. 5. Lines are drawn from the cumulative energy point $(i, e(i))$ to the future points $(j, e(j))$ for all $j>i$ and the one with the minimum slope is chosen which has the index $j^{*}$. The corresponding slope is the allocated power for all frames between $i$
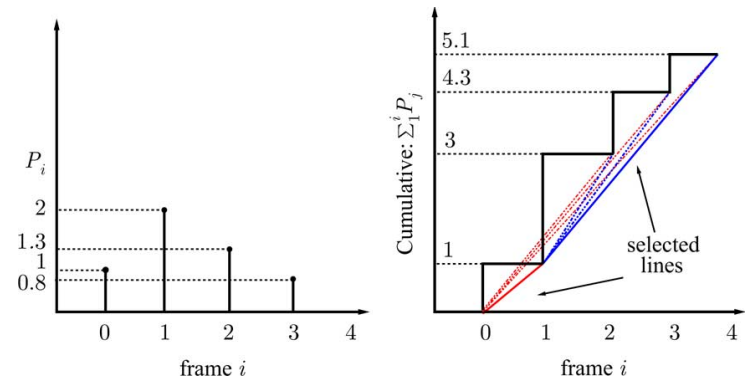

Fig. 5. Operation of the algorithm that finds optimal power management.

and $j^{*}$. In the depicted example, there are $L=4$ frames and $P_{1}=1, P_{2}=2, P_{3}=1.3$, and $P_{4}=0.8$. We start with calculating the slopes by connecting $(0,0)$ to $(j, e(j))$ for $j>0$, which are $\frac{1}{1}, \frac{1+2}{2}, \frac{1+2+1.3}{3}$, and $\frac{1+2+1.3+0.8}{4}$. We observe that the minimum slope is obtained by connecting $(0,0)$ to $(1, e(1))$. Hence, the optimal power level in frame 1 is $Q_{1}^{*}=1$. We next determine power levels for $i>1$, which are larger than $Q_{1}^{*}=1$. Proceeding similarly, by connecting $(1, e(1))$ to $(j, e(j))$ for $j>1$, the minimum slope is obtained by connecting $(1, e(1))$ and $(4, e(4))$, and hence, the optimal power levels are $Q_{i}^{*}=$ $\frac{2+1.3+0.8}{3}=1.37$ for $2 \leq i \leq 4$. There are $K=2$ constant power bands where $L_{1}=0, L_{2}=1$, and $L_{3}=4$.

\section{NUMERICAL RESULTS}

The optimum power management algorithm takes the arrival rate vector $\left[P_{1}, \ldots, P_{L}\right]$ and outputs the optimal power vector $\left[Q_{1}^{*}, \ldots, Q_{L}^{*}\right]$. We let the arrival rates of energy in all frames $P_{i}$ follow an i.i.d. exponential distribution. A benchmark algorithm is simply no power management algorithm, i.e., $Q_{i}=P_{i}$. In this simple scheme, the energy arrival rate in each frame is taken as the communication power in that frame. This scheme yields an average throughput

$$
T_{l b}=\frac{1}{L} \sum_{i=1}^{L} \frac{1}{2} \log \left(1+P_{i}\right)
$$

which is a lower bound. However, if the designer has the information of arrival rates in future frames, then the optimal power management algorithm can improve the average throughput. It is clear that an upper bound for the average throughput is

$$
T_{u b}=\frac{1}{2} \log \left(1+\frac{1}{L} \sum_{i=1}^{L} P_{i}\right)
$$

which assumes that $\sum_{i=1}^{L} P_{i}$ is available at the beginning, and therefore can be spread evenly over all time.

The comparison of the performances of the optimal power management algorithm with the upper bound $T_{u b}$ and the lower bound $T_{l b}$ (no power management) is given in Fig. 6 for $L=20$ frames. We observe that as the variance of the arrival rates increases, the advantage of optimal power management becomes more apparent with respect to no power management. Another observation is that the difference between the upper bound and the average throughput with optimal power management also increases as the standard deviation of the arrival 


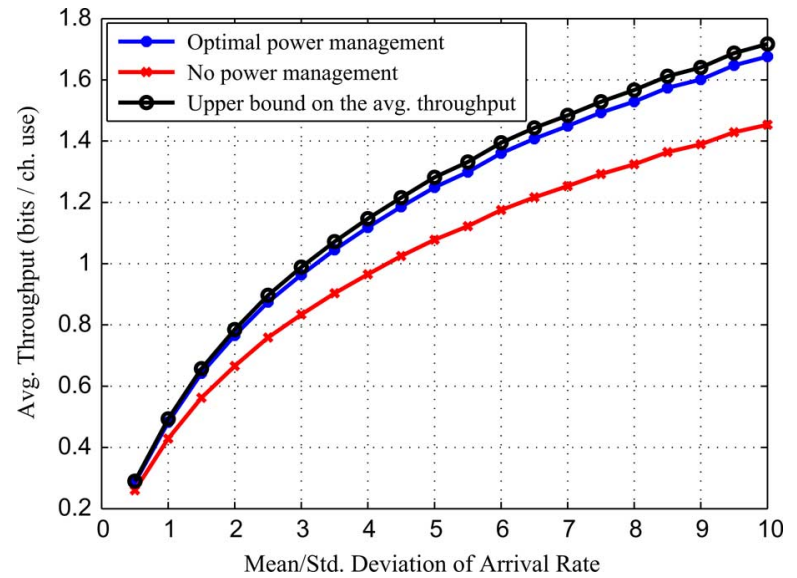

Fig. 6. Average throughput versus mean/standard deviation of the arrival rate for $L=20$ frames.

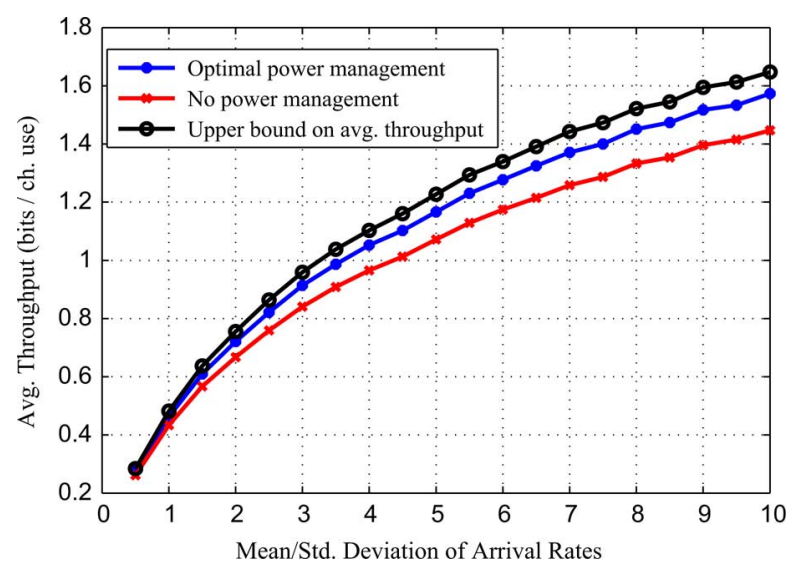

Fig. 7. Average throughput versus mean/standard deviation of the arrival rate for $L=5$ frames.

rate is increased. Hence, the causality constraint becomes more restrictive as the variation in the arrival rate is increased.

The comparison of the performances of the optimal power management with the upper bound $T_{u b}$ and the lower bound $T_{l b}$ (no power management) is given in Fig. 7 for a $L=5$ frame system. We observe that the upper bound and the average throughput with the optimal power management scheme are strictly smaller for $L=20$ frames. This difference becomes more apparent when the variance of the arrival rates is higher. The upper bound has smaller value because arrival rates cannot be averaged sufficiently in $L=5$ frames. Moreover, since $L=5$ frames is not long enough to react to peaks in the arrival rate by saving and spreading the energy for future frames, average throughput with the optimal power management scheme is smaller in this case.

\section{CONCLUSION}

We established the capacity of the AWGN channel under stochastic energy harvesting where an unlimited sized battery buffers communication energy between an uncontrolled recharge process and the transmitter. This nature of the energy arrivals yields an unprecedented power constraint on each code symbol. Remarkably, communication can be performed at the capacity of the average power-constrained AWGN channel. We first presented a save-and-transmit scheme in which data transmission occurs in two phases. In the first phase, energy is collected and in the second phase, data are transmitted. Next, we provided an alternative best-effort-transmit scheme that achieves the capacity without utilizing an initial saving phase. Finally, we extended our model to time-varying recharge rates in large time scales. We obtained optimal offline power management for maximum average throughput and illustrated its operation geometrically.

\section{APPENDIX A}

\section{PROOF OF LEMMA 1}

In view of the instantaneous energy constraints in (1) in each channel use, in order to prove the statement of the lemma, we need to show that for any $\epsilon>0$ and sufficiently large $n$

$$
\operatorname{Pr}\left(\bigcup_{k=1}^{n}\left\{\sum_{i=1}^{k} E_{i}<\sum_{i=1}^{k} X_{i}^{2}\right\}\right) \leq \epsilon .
$$

In the saving phase, $X_{i}=0$ for $i=1, \ldots, h(n)$. Hence, the events $\left\{\sum_{i=1}^{k} E_{i}<\sum_{i=1}^{k} X_{i}^{2}\right\}=\emptyset$ for the saving phase. For convenience, we use the index $s$ for the saving phase, i.e., $s=1, \ldots, h(n)$ and the index $t$ for the transmission phase, i.e., $t=1, \ldots, n-h(n)$. We have $E\left[E_{t}\right]=P, E\left[X_{t}^{2}\right]=P_{\text {avg }}$ and $P_{\text {avg }}<P$. Note that $E_{s}, s=1, \ldots, h(n)$ and $E_{t}, t=$ $1, \ldots, n-h(n)$, are independent. Thus, we need to show that for sufficiently large $n$

$$
\operatorname{Pr}\left(\bigcup_{k=1}^{n-h(n)}\left\{\sum_{s=1}^{h(n)} E_{s}+\sum_{t=1}^{k} E_{t}<\sum_{t=1}^{k} X_{t}^{2}\right\}\right) \leq \epsilon .
$$

By the strong law of large numbers [18], we have as $n \rightarrow \infty$

$$
\frac{1}{n-h(n)}\left(\sum_{t=1}^{n-h(n)} E_{t}-\sum_{t=1}^{n-h(n)} X_{t}^{2}\right) \rightarrow P-P_{\text {avg }}, \text { w.p. } 1
$$

since $n-h(n) \rightarrow \infty$ as $n \rightarrow \infty$. Then, (23) implies for any $\delta>0$

$$
\lim _{m \rightarrow \infty} \operatorname{Pr}\left(\bigcup_{k=m}^{\infty}\left\{\left|\frac{\sum_{t=1}^{k} E_{t}-X_{t}^{2}}{k}-\left(P-P_{\text {avg }}\right)\right|>\delta\right\}\right)=0 .
$$

Choosing $\delta=P-P_{\text {avg }}$, there exists sufficiently large $k_{0}$ such that

$$
\operatorname{Pr}\left(\bigcup_{k=k_{0}}^{\infty}\left\{\sum_{t=1}^{k} E_{t}<\sum_{t=1}^{k} X_{t}^{2}\right\}\right)<\epsilon^{\prime}
$$

Therefore, since $\sum_{s=1}^{h(n)} E_{s} \geq 0$, for all $n-h(n) \geq k_{0},(25)$ implies

$$
\operatorname{Pr}\left(\bigcup_{k=k_{0}}^{n-h(n)}\left\{\sum_{s=1}^{h(n)} E_{s}+\sum_{t=1}^{k} E_{t}<\sum_{t=1}^{k} X_{t}^{2}\right\}\right)<\epsilon^{\prime}
$$


In order to reach (22), it remains to show that $\operatorname{Pr}\left(\bigcup_{k=1}^{k_{0}-1}\left\{\sum_{s=1}^{h(n)} E_{s}+\sum_{t=1}^{k} E_{t}<\sum_{t=1}^{k} X_{t}^{2}\right\}\right) \quad$ can be made arbitrarily small by selecting $n$ sufficiently large. We first apply the union bound

$$
\begin{aligned}
\operatorname{Pr}\left(\bigcup_{k=1}^{k_{0}-1}\left\{\sum_{s=1}^{h(n)} E_{s}+\sum_{t=1}^{k} E_{t}<\sum_{t=1}^{k} X_{t}^{2}\right\}\right) \\
\leq \sum_{k=1}^{k_{0}-1} \operatorname{Pr}\left(\left\{\sum_{s=1}^{h(n)} E_{s}+\sum_{t=1}^{k} E_{t}<\sum_{t=1}^{k} X_{t}^{2}\right\}\right)
\end{aligned}
$$

By the weak law of large numbers [18], for every $\kappa>0, \delta>0$, and sufficiently large $n$

$$
\operatorname{Pr}\left(\left|\frac{1}{h(n)} \sum_{s=1}^{h(n)} E_{s}-P\right|>\delta\right)<\kappa
$$

Define the event $A_{\delta, n}=\left\{\left|\frac{1}{h(n)} \sum_{s=1}^{h(n)} E_{s}-P\right|>\delta\right\}$. Conditioning on $A_{\delta, n}$ and using the law of total probability, and in view of the independence of $E_{s}$ and $E_{t}$, we have for any $\kappa>0$, $\delta>0$, and sufficiently large $n$

$$
\begin{aligned}
& \operatorname{Pr}\left(\left\{\sum_{s=1}^{h(n)} E_{s}+\sum_{t=1}^{k} E_{t}<\sum_{t=1}^{k} X_{t}^{2}\right\}\right) \\
& =\operatorname{Pr}\left(\left\{\sum_{s=1}^{h(n)} E_{s}+\sum_{t=1}^{k} E_{t}<\sum_{t=1}^{k} X_{t}^{2} \mid A_{\delta, n}^{c}\right\}\right) \operatorname{Pr}\left(A_{\delta, n}^{c}\right) \\
& +\operatorname{Pr}\left(\left\{\sum_{s=1}^{h(n)} E_{s}+\sum_{t=1}^{k} E_{t}<\sum_{t=1}^{k} X_{t}^{2} \mid A_{\delta, n}\right\}\right) \operatorname{Pr}\left(A_{\delta, n}\right) \\
& \leq \operatorname{Pr}\left(\left\{h(n)(P-\delta)+\sum_{t=1}^{k} E_{t}<\sum_{t=1}^{k} X_{t}^{2}\right\}\right)+\kappa \\
& \leq \epsilon^{\prime \prime} .
\end{aligned}
$$

Note that neither $\sum_{t=1}^{k} E_{t}$ nor $\sum_{t=1}^{k} X_{t}^{2}$ depends on $n$. Using (31) in (27), for sufficiently large $n$, we have

$$
\operatorname{Pr}\left(\bigcup_{k=1}^{k_{0}-1}\left\{\sum_{s=1}^{h(n)} E_{s}+\sum_{t=1}^{k} E_{t}<\sum_{t=1}^{k} X_{t}^{2}\right\}\right) \leq \epsilon^{\prime \prime \prime}
$$

Then, using (26) and (32), combined with the union bound, we get

$$
\operatorname{Pr}\left(\bigcup_{k=1}^{n-h(n)}\left\{\sum_{s=1}^{h(n)} E_{s}+\sum_{t=1}^{k} E_{t}<\sum_{t=1}^{k} X_{t}^{2}\right\}\right) \leq \epsilon^{\prime}+\epsilon^{\prime \prime \prime} \triangleq \epsilon
$$

which is what we need in (22).

\section{APPENDIX B}

Calculation of (8) AND (9)

For convenience, we use $s=1, \ldots, h(n)$ for the saving phase and $t=1, \ldots, n-h(n)$ for the transmission phase as in Appendix A. Since $X_{s}=0$ for $s=1, \ldots, h(n)$, we have

$$
\operatorname{Pr}\left(\sum_{i=1}^{n} X_{i}^{2}>\sum_{i=1}^{n} E_{i}\right)=\operatorname{Pr}\left(\sum_{t=1}^{n-\sqrt{n}} Z_{t}>\sum_{s=1}^{\sqrt{n}} E_{s}\right)
$$

where $Z_{t}=X_{t}^{2}-E_{t}$ and thus $E\left[Z_{t}\right]=0$ for $t=1, \ldots, n-\sqrt{n}$. Let the event $B_{\delta, n}$ be $B_{\delta, n} \triangleq\left\{\left|\frac{1}{\sqrt{n}} \sum_{s=1}^{\sqrt{n}} E_{s}-P\right|>\delta\right\}$. By the law of total probability, we have

$$
\begin{aligned}
\operatorname{Pr}\left(\sum_{t=1}^{n-\sqrt{n}} Z_{t}>\sum_{s=1}^{\sqrt{n}} E_{s}\right) & \\
= & \operatorname{Pr}\left(\sum_{t=1}^{n-\sqrt{n}} Z_{t}>\sum_{s=1}^{\sqrt{n}} E_{s} \mid B_{\delta, n}\right) \operatorname{Pr}\left(B_{\delta, n}\right) \\
& +\operatorname{Pr}\left(\sum_{t=1}^{n-\sqrt{n}} Z_{t}>\sum_{s=1}^{\sqrt{n}} E_{s} \mid B_{\delta, n}^{c}\right) \operatorname{Pr}\left(B_{\delta, n}^{c}\right) .
\end{aligned}
$$

Note that we have

$$
\begin{array}{r}
\operatorname{Pr}\left(\sum_{t=1}^{n-\sqrt{n}} Z_{t}>\sqrt{n}(P+\delta)\right) \\
\leq \operatorname{Pr}\left(\sum_{t=1}^{n-\sqrt{n}} Z_{t}>\sum_{s=1}^{\sqrt{n}} E_{s} \mid A_{\delta, n}^{c}\right) \\
\leq \operatorname{Pr}\left(\sum_{t=1}^{n-\sqrt{n}} Z_{t}>\sqrt{n}(P-\delta)\right) .
\end{array}
$$

From the central limit theorem [18], for $n$ i.i.d. samples of a random variable $D_{i}$ with zero mean and variance $d^{2}$, we have

$$
\lim _{n \rightarrow \infty} \operatorname{Pr}\left(\frac{1}{\sqrt{n}} \sum_{i=1}^{n} D_{i}>x\right)=\Phi\left(\frac{x}{d}\right)
$$

where $\Phi(x)=\int_{x}^{\infty} \frac{1}{\sqrt{2 \pi}} e^{-\frac{\tau^{2}}{2}} d \tau$. In view of the fact that $\frac{1}{\sqrt{n}} \sum_{i=1}^{\sqrt{n}} D_{i} \rightarrow 0$ almost surely as $n \rightarrow \infty$, we have

$$
\lim _{n \rightarrow \infty} \operatorname{Pr}\left(\frac{1}{\sqrt{n}} \sum_{i=1}^{n-\sqrt{n}} D_{i}>x\right)=\Phi\left(\frac{x}{d}\right) .
$$

Applying (39) for $Z_{t}, t=1, \ldots, n-\sqrt{n}$, we get

$$
\lim _{n \rightarrow \infty} \operatorname{Pr}\left(\sum_{t=1}^{n-\sqrt{n}} Z_{t}>\sqrt{n} P\right)=\Phi\left(\frac{P}{a}\right)
$$

where we assume that the variance of $Z_{t}$ is finite and equal to $a^{2}$. We can have this, for instance, when $E_{t}$ has finite variance, $\sigma_{E}^{2}$. In this case, as $X_{t}$ is Gaussian and independent of $E_{t}$, we have $a^{2} \triangleq E\left[Z_{t}^{2}\right]=\sigma_{E}^{2}+2 P^{2}$. As a consequence, 
$\lim _{n \rightarrow \infty} \operatorname{Pr}\left(\sum_{t=1}^{n-\sqrt{n}} Z_{t}^{2}>\sqrt{n}(P \pm \delta)\right)=\Phi\left(\frac{P \pm \delta}{a}\right)$. Since $\Phi(x)$ is continuous in $x$, in view of (36) and (37), we have

$$
\lim _{\delta \rightarrow 0^{+}} \lim _{n \rightarrow \infty} \operatorname{Pr}\left(\sum_{t=1}^{n-\sqrt{n}} Z_{t}^{2}>\sum_{s=1}^{\sqrt{n}} E_{s} \mid A_{\delta, n}^{c}\right)=\Phi\left(\frac{P}{a}\right) .
$$

By the weak law of large numbers [18], $\lim _{n \rightarrow \infty} \operatorname{Pr}\left(A_{\delta, n}^{c}\right)=1$ for all $\delta>0$ and evaluating (35) as $\delta \rightarrow 0^{+}$, we get

$$
\begin{aligned}
\lim _{n \rightarrow \infty} \operatorname{Pr}\left(\sum_{i=1}^{n} X_{i}^{2}>\sum_{i=1}^{n} E_{i}\right) & =\lim _{n \rightarrow \infty} \operatorname{Pr}\left(\sum_{t=1}^{n-\sqrt{n}} Z_{t}>\sqrt{n} P\right) \\
& =\Phi\left(\frac{P}{a}\right)
\end{aligned}
$$

which is (8).

When $h(n)=\log (n)$, as in the aforementioned derivation, this probability becomes

$$
\begin{aligned}
\lim _{n \rightarrow \infty} \operatorname{Pr}\left(\sum_{i=1}^{n} X_{i}^{2}>\sum_{i=1}^{n} E_{i}\right) \\
=\lim _{n \rightarrow \infty} \operatorname{Pr}\left(\sum_{t=1}^{n-\log (n)} Z_{t}>\log (n) P\right) \\
=\lim _{n \rightarrow \infty} \Phi\left(\frac{P}{a} \sqrt{\frac{\log (n)}{\sqrt{n}}}\right) \\
=\Phi(0) \\
=\frac{1}{2}
\end{aligned}
$$

which is (9).

\section{APPENDIX C}

ProOF OF LEMMA 2

We need to show the following result for sufficiently large $n$ :

$$
\operatorname{Pr}\left(\bigcup_{k=1}^{n}\left\{\sum_{i=1}^{k} E_{i}<\sum_{i=1}^{k} X_{i}^{2}\right\}\right) \leq \epsilon .
$$

We have again $\left\{\sum_{i=1}^{k} E_{i}<\sum_{i=1}^{k} X_{i}^{2}\right\}=\emptyset$ for $k=$ $1, \ldots, h(n)$. As $E\left[X_{i}^{2}\right]=E\left[E_{i}\right]=P$ in the transmission phase, we cannot proceed by using the strong law of large numbers. Recall that in the proof of Lemma 1, the strong law of large numbers is invoked in (25) by choosing $\delta=P-P_{\text {avg }}$; however, in this case, since $P-P_{\text {avg }}=0, \delta=P-P_{\text {avg }}=0$ is not allowed as a selection. Our proof for the $P=P_{\text {avg }}$ case uses a stronger version of Marcinkiewicz-Zygmund type strong law of large numbers that is originally proved in [19]. In particular, we use [19, Corollary 2.16], which we state next for completeness.

Theorem 4 (Corollary 2.16 in [19]): Let $\left\{X_{i}\right\}$ be a sequence of i.i.d. random variables with $E\left[X_{i}\right]=0$ and let $\left\{a_{n i}, 1 \leq i \leq n, n \geq 1\right\}$ be a triangular array of constants satisfying $A_{\alpha}=\limsup _{n \rightarrow \infty} A_{\alpha, n}<\infty$ where $A_{\alpha, n}=\frac{1}{n} \sum_{i=1}^{n}\left|a_{n i}\right|^{\alpha}$ for some $1<\alpha \leq 2$. Let $T_{n}=\sum_{i=1}^{n} a_{n i} X_{i}, n \geq 1$, and let $b_{n}=n^{\frac{1}{\alpha}}(\log (n))^{\frac{1}{\gamma}}$. Moreover, for some $h>0$ and $\gamma>0$, we assume $E\left[e^{h|X|^{\gamma}}\right]<\infty$. Then

$$
\lim _{n \rightarrow \infty} \frac{\left|T_{n}\right|}{b_{n}}=0, \quad \text { a.s. }
$$

As in Appendix A, we use the index $s$ for the saving phase, i.e., $s=1, \ldots, h(n)$ and the index $t$ for the transmission phase, i.e., $t=1, \ldots, n-h(n)$. We start by noting that the condition in (48) is equivalent to the following for all $\epsilon>0$ and sufficiently large $n$ :

$$
\operatorname{Pr}\left(\bigcup_{k=1}^{n-h(n)}\left\{\sum_{t=1}^{k} Z_{t}>\sum_{s=1}^{h(n)} E_{s}\right\}\right) \leq \epsilon
$$

where $Z_{t}=X_{t}^{2}-E_{t}$. Note that the random variables $\left\{E_{s}\right\}_{s=1}^{h(n)}$ are independent of $\left\{Z_{t}\right\}_{t=1}^{n-h(n)}$ and $E\left[Z_{t}\right]=0$, while $E\left[E_{i}\right]=$ $P$. In order to show (50), we replace $T_{k}=\sum_{t=1}^{k} Z_{t}$ where we take the triangular array in Theorem 4 as $a_{n i}=1$. Note that this agrees with $A_{\alpha}<\infty$ requirement as this selection leads to $A_{\alpha}=1$ for any $\alpha$.

By the weak law of large numbers [18], for every $\epsilon>0$, $\delta>0$, and sufficiently large $n$

$$
\operatorname{Pr}\left(\left|\frac{1}{h(n)} \sum_{s=1}^{h(n)} E_{s}-P\right|>\delta\right)<\epsilon .
$$

Define the event $C_{\delta, n}=\left\{\left|\frac{1}{h(n)} \sum_{s=1}^{h(n)} E_{s}-P\right|>\delta\right\}$. Conditioning on $C_{\delta, n}$ and using the law of total probability in a similar fashion to the corresponding steps in Appendices A and B, we have for any $\epsilon>0, \delta>0$, and sufficiently large $n$

$$
\begin{aligned}
& \operatorname{Pr}\left(\bigcup_{k=1}^{n-h(n)}\left\{\sum_{t=1}^{k} Z_{t}>\sum_{s=1}^{h(n)} E_{s}\right\}\right) \\
& \quad \leq \operatorname{Pr}\left(\bigcup_{k=1}^{n-h(n)}\left\{\sum_{t=1}^{k} Z_{t}>h(n)(P-\delta)\right\}\right)+\epsilon .
\end{aligned}
$$

Therefore, we need to show that for any $\delta>0, \epsilon^{\prime}>0$, and sufficiently large $n$

$$
\operatorname{Pr}\left(\bigcup_{k=1}^{n-h(n)}\left\{\sum_{t=1}^{k} Z_{t}>h(n)(P-\delta)\right\}\right)<\epsilon^{\prime} .
$$

Now, we let $h(n)=n^{\frac{1}{\alpha}}(\log (n))^{\frac{1}{\gamma}}$ for some $1<\alpha \leq 2$ and $\gamma>0$. Moreover, we note that $E\left[e^{\left|Z_{t}\right|^{\gamma}}\right]<\infty$ for $0<$ $\gamma<1$. To see this, we first note $Z_{t}=X_{t}^{2}-E_{t}$ and $X_{t}^{2}>0$, $E_{t}>0$. Hence, we get $\left|Z_{t}\right|^{\gamma} \leq X_{t}^{2 \gamma}+E_{t}^{\gamma}$ for $0<\gamma<1$ and hence $e^{\mid Z_{t} t^{\prime \gamma}} \leq e^{X_{t}^{2 \gamma}} e^{E_{t}^{\gamma}}$. Since $X_{t}$ is zero-mean Gaussian with variance $P, E\left[e^{X_{t}^{2 \gamma}}\right]<\infty$ for $0<\gamma<1$. That is, the hypothesis $E\left[e^{E_{t}^{\gamma}}\right]<\infty$ implies $E\left[e^{\left|Z_{t}\right|^{\gamma}}\right]<\infty$, which is a requirement for Theorem 4 . Therefore, by Theorem 4 , we have

$$
\lim _{n \rightarrow \infty} \frac{\left|\sum_{t=1}^{n} Z_{t}\right|}{h(n)}=0, \quad \text { w.p.1. }
$$


Equation (54) implies for any $\bar{\delta}>0$

$$
\lim _{n \rightarrow \infty} \operatorname{Pr}\left(\bigcup_{k=n}^{\infty}\left\{\left|\sum_{t=1}^{k} Z_{t}\right|>h(k) \bar{\delta}\right\}\right)=0 .
$$

Therefore, for any $\epsilon>0$, there exists sufficiently large $k_{0}$ such that [cf., (25)]

$$
\operatorname{Pr}\left(\bigcup_{k=k_{0}}^{\infty}\left\{\left|\sum_{t=1}^{k} Z_{t}\right|>h(k) \bar{\delta}\right\}\right)<\epsilon .
$$

In particular, we have for $n-h(n) \geq k_{0}$ and $\bar{\delta}=P-\delta$

$$
\operatorname{Pr}\left(\bigcup_{k=k_{0}}^{n-h(n)}\left\{\left|\sum_{t=1}^{k} Z_{t}\right|>h(n)(P-\delta)\right\}\right)<\epsilon
$$

where we use the fact that $h(k)<h(n)$ for all $k=k_{0}, \ldots, n-$ $h(n)$. In order to show (53), it remains to prove that for sufficiently large $n$

$$
\operatorname{Pr}\left(\bigcup_{k=1}^{k_{0}-1}\left\{\left|\sum_{t=1}^{k} Z_{t}\right|>h(n)(P-\delta)\right\}\right)<\epsilon .
$$

Using the union bound, we have

$$
\begin{aligned}
\operatorname{Pr}\left(\bigcup_{k=1}^{k_{0}-1}\right. & \left.\left\{\left|\sum_{t=1}^{k} Z_{t}\right|>h(n)(P-\delta)\right\}\right) \\
& \leq \sum_{k=1}^{k_{0}-1} \operatorname{Pr}\left(\left|\sum_{t=1}^{k} Z_{t}\right|>h(n)(P-\delta)\right)
\end{aligned}
$$

We note that $\operatorname{Pr}\left(\left|\sum_{t=1}^{k} Z_{t}\right|>h(n)(P-\delta)\right) \rightarrow 0$ as $h(n) \rightarrow$ $\infty$. Hence, (58) holds for sufficiently large $n$.

Therefore, under the hypothesis of Lemma 2, probability of energy shortage goes to zero as $n$ gets large. This establishes Lemma 2.

\section{APPENDIX D}

\section{PROOF OF THEOREM 3}

First, we observe that the transmit power vector $\mathrm{Q}^{*}$ defined in (17) and (18) has monotonically increasing entries, i.e., $Q_{1}^{*} \leq$ $Q_{2}^{*} \leq \cdots \leq Q_{L}^{*}$. This is true, because otherwise we could construct a line with a smaller slope that connects two energy arrival points and this would contradict the definition of the algorithm in (17) and (18).

Let $\left\{L_{1}, \ldots, L_{K+1}\right\}$ be the indices of constant power bands $\left\{L_{k}-L_{k+1}\right\}$ and let $\mathbf{Q}=\left[Q_{1}, \ldots, Q_{L}\right]$ be any feasible power vector. We will show that $\mathbf{Q}^{*} \preceq \mathbf{Q}$ by verifying that all of the conditions in (14) are satisfied. Note that the condition in (15) is satisfied by definition of feasibility.
Since the algorithm produces monotone increasing powers, $Q_{(j)}^{*}=Q_{L}^{*}, j=1, \ldots, L-L_{K}$. In particular, $Q_{(1)}^{*}=\max _{i} Q_{i}^{*}=Q_{L}^{*}$ and as $\mathbf{Q}$ is feasible, we have

$$
\sum_{j=1}^{L_{k}} Q_{j} \leq \sum_{j=1}^{L_{k}} Q_{j}^{*}, \quad k=1, \ldots, K
$$

Moreover, by feasibility, we have the equality $\sum_{j=1}^{L} Q_{j}=$ $\sum_{j=1}^{L} Q_{j}^{*}$. Hence, (60) and the equality implies

$$
\sum_{j=L_{k}+1}^{L} Q_{j}^{*} \leq \sum_{j=L_{k}+1}^{L} Q_{j}, \quad k=1, \ldots, K .
$$

Applying (61) at $k=K$

$$
Q_{(1)}^{*}=\frac{\sum_{j=L_{K}+1}^{L} Q_{j}^{*}}{L-L_{K}} \leq \frac{\sum_{j=L_{K}+1}^{L} Q_{j}}{L-L_{K}} \leq \frac{\sum_{j=1}^{L-L_{K}} Q_{(j)}}{L-L_{K}}
$$

By rearranging the terms

$$
\left(L-L_{K}\right) Q_{(1)}^{*}=\sum_{j=1}^{L-L_{K}} Q_{(j)}^{*} \leq \sum_{j=1}^{L-L_{K}} Q_{(j)} .
$$

Since $Q_{(j)}$ is ordered and $Q_{(j)}^{*}=Q_{(1)}^{*}$ for $j=1, \ldots, L-L_{K}$, we have the following:

$$
\sum_{j=1}^{\ell} Q_{(j)}^{*} \leq \sum_{j=1}^{\ell} Q_{(j)}, \quad \ell=1, \ldots, L-L_{K} .
$$

The remaining conditions are verified similarly. Again, since the algorithm yields monotone increasing powers, for $j=L-$ $L_{K}+1, \ldots, L-L_{K-1}, Q_{(j)}^{*}=Q_{\left(L-L_{K}+1\right)}^{*}$. By applying (61) at $k=K-2$, we have

$$
\begin{aligned}
\left(L_{K}\right. & \left.-L_{K-1}\right) Q_{\left(L-L_{K}+1\right)}^{*}+\left(L-L_{K}\right) Q_{(1)}^{*} \\
& =\sum_{j=1}^{L-L_{K-1}} Q_{(j)}^{*} \\
& \leq \sum_{j=1}^{L-L_{K-1}} Q_{(j)} .
\end{aligned}
$$

Then, we must have

$$
\sum_{j=1}^{\ell} Q_{(j)}^{*} \leq \sum_{j=1}^{\ell} Q_{(j)}, \quad \ell=L-L_{K}+1, \ldots, L-L_{K-1} .
$$

Repeating this argument, we verify all conditions required to get (14) for $\mathbf{Q}^{*}$ and $\mathbf{Q}$ in places of $\mathbf{x}$ and $\mathbf{y}$.

\section{ACKNOWLEDGMENT}

We acknowledge anonymous reviewers whose careful reading and detailed comments improved the content and the presentation of our work. 


\section{REFERENCES}

[1] O. Ozel and S. Ulukus, "Information theoretic analysis of an energy harvesting communication system," in Proc. 21st Int. Symp. Pers. Indoor Mobile Radio Commun. Workshop, Sep. 2010, pp. 330-335.

[2] J. Lei, R. Yates, and L. Greenstein, "A generic model for optimizing single-hop transmission policy of replenishable sensors," IEEE Trans. Wireless Commun., vol. 8, no. 2, pp. 547-551, Feb. 2009.

[3] M. Gatzianas, L. Georgiadis, and L. Tassiulas, "Control of wireless networks with rechargeable batteries," IEEE Trans. Wireless Commun., vol. 9, no. 2, pp. 581-593, Feb. 2010.

[4] V. Sharma, U. Mukherji, V. Joseph, and S. Gupta, "Optimal energy management policies for energy harvesting sensor nodes," IEEE Trans. Wireless Commun., vol. 9, no. 4, pp. 1326-1336, Apr. 2010.

[5] V. Sharma, U. Mukherji, and V. Joseph, "Efficient energy management policies for networks with energy harvesting sensor nodes," in Proc. 46th Annu. Allerton Conf. Commun., Control, Comput., Sep. 2008, pp. 375-383.

[6] J. Yang and S. Ulukus, "Transmission completion time minimization in an energy harvesting system," in Proc. 44th Annu. Conf. Inf. Sci. Syst., Mar. 2010, pp. 1-6.

[7] J. Yang and S. Ulukus, "Optimal packet scheduling in an energy harvesting communication system," IEEE Trans. Commun., vol. 60, no. 1, pp. 220-230, Jan. 2012.

[8] C. K. Ho and R. Zhang, "Optimal energy allocation for wireless communications powered by energy harvesters," in Proc. IEEE Int. Symp. Inf. Theory, Jun. 2010, pp. 2368-2372.

[9] O. Ozel, K. Tutuncuoglu, J. Yang, S. Ulukus, and A. Yener, “Transmission with energy harvesting nodes in fading wireless channels: Optimal policies," IEEE J. Sel. Areas Commun., vol. 29, no. 8, pp. 1732-1743, Sep. 2011.

[10] K. Tutuncuoglu and A. Yener, "Optimum transmission policies for battery limited energy harvesting systems," IEEE Trans. Wireless Commun., vol. 11, no. 3, pp. 1180-1189, Mar. 2012.

[11] J. Yang, O. Ozel, and S. Ulukus, "Broadcasting with an energy harvesting rechargeable transmitter," IEEE Trans. Wireless Commun., vol. 11, no. 2, pp. 571-583, Feb. 2012.

[12] M. A. Antepli, E. Uysal-Biyikoglu, and H. Erkal, "Optimal packet scheduling on an energy harvesting broadcast link," IEEE J. Sel. Areas Commun., vol. 29, no. 8, pp. 1721-1731, Sep. .2011.

[13] J. G. Smith, "The information capacity of amplitude and variance-constrained scalar Gaussian channels," Inf. Control, vol. 18, pp. 203-219, Apr. 1971.

[14] S. Shamai and I. Bar-David, "The capacity of average and peak-powerlimited quadrature Gaussian channels," IEEE Trans. Inf. Theory, vol. 41, no. 4, pp. 1060-1071, Jul. 1995.

[15] M. Raginsky, "On the information capacity of Gaussian channels under small peak power constraints," in Proc. 46th Annu. Allerton Conf. Commun., Control, Comput., Sep. 2008, pp. 286-293.

[16] O. Ozel and S. Ulukus, "AWGN channel under time-varying amplitude constraints with causal information at the transmitter," in Proc. 45th Asilomar Conf. Signals, Syst. Comput., Nov. 2011, pp. 373-377.

[17] S. Verdu and T. S. Han, "A general formula for channel capacity," IEEE Trans. Inf. Theory, vol. 40, no. 4, pp. 1147-1157, Jul. 1994.

[18] G. Grimmet and D. Stirzaker, Probability and Random Processes. New York: Oxford Univ. Press, 2001.

[19] G. H. Cai, "Strong laws for weighted sums of i.i.d random variables," Electron. Res. Announcements Amer. Math. Soc., vol. 12, pp. 29-36, Mar. 2008.
[20] T. M. Cover and J. Thomas, Elements of Information Theory. Hoboken, NJ: Wiley, 2006.

[21] G. Caire and S. Shamai, "On the capacity of some channels with channel state information," IEEE Trans. Inf. Theory, vol. 45, no. 6, pp. 2007-2019, Sep. 1999.

[22] E. Jorswieck and H. Boche, Majorization and Matrix Monotone Functions in Wireless Communication. Hanover, MA: Now Publishers Inc., 2007.

[23] M. Zafer and E. Modiano, "A calculus approach to energy-efficient data transmission with quality-of-service constraints," IEEE/ACM Trans. Netw., vol. 17, no. 3, pp. 898-911, Jun. 2009.

Omur Ozel (S'90) received the B.Sc. and the M.S. degrees with honors in electrical and electronics engineering from the Middle East Technical University (METU), Ankara, Turkey, in June 2007 and July 2009, respectively. Since August 2009, he has been a graduate research assistant at the University of Maryland College Park, working towards Ph.D. degree in electrical and computer engineering. His research focuses on information and network theoretical aspects of energy harvesting communication systems. He is a graduate student member of IEEE.

Sennur Ulukus (S'90-M'98) is a Professor of Electrical and Computer Engineering at the University of Maryland at College Park, where she also holds a joint appointment with the Institute for Systems Research (ISR). Prior to joining UMD, she was a Senior Technical Staff Member at AT\&T Labs-Research. She received her Ph.D. degree in Electrical and Computer Engineering from Wireless Information Network Laboratory (WINLAB), Rutgers University, and B.S. and M.S. degrees in Electrical and Electronics Engineering from Bilkent University. Her research interests are in wireless communication theory and networking, network information theory for wireless communications, signal processing for wireless communications, information-theoretic physical-layer security, and energy-harvesting communications.

Dr. Ulukus received the 2003 IEEE Marconi Prize Paper Award in Wireless Communications, the 2005 NSF CAREER Award, and the 2010-2011 ISR Outstanding Systems Engineering Faculty Award. She served as an Associate Editor for the IEEE TRANSACTIONS ON INFORMATION THEORY between 2007-2010, as an Associate Editor for the IEEE TRANSACTIONS ON COMMUNICATIONS between 2003-2007, as a Guest Editor for the Journal of Communications and Networks for the special issue on energy harvesting in wireless networks, as a Guest Editor for the IEEE TRANSACTIONS ON INFORMATION THEORY for the special issue on interference networks, as a Guest Editor for the IEEE JOURNAL ON SELECTED AREAS IN COMMUNICATIONS for the special issue on multiuser detection for advanced communication systems and networks. She served as the TPC co-chair of the Communication Theory Symposium at the 2007 IEEE Global Telecommunications Conference, the Medium Access Control (MAC) Track at the 2008 IEEE Wireless Communications and Networking Conference, the Wireless Communications Symposium at the 2010 IEEE International Conference on Communications, the 2011 Communication Theory Workshop, the Physical-Layer Security Workshop at the 2011 IEEE International Conference on Communications, the Physical-Layer Security Workshop at the 2011 IEEE Global Telecommunications Conference. She was the Secretary of the IEEE Communication Theory Technical Committee (CTTC) in 2007-2009. 